\title{
Necroptosis-associated LncRNA markers for late survival prediction, immune and gene mutation analysis in ovarian cancer
}

weisen fan ( $\sim 2511783676 @ q q . c o m)$

Shandong University of Traditional Chinese Medicine https://orcid.org/0000-0001-6114-5586 yinghua qi

Shandong University of Traditional Chinese Medicine Affiliated Hospital

zhongqi fan

Shandong University of Traditional Chinese Medicine

haoying meng

Shandong University of Traditional Chinese Medicine

rumeng yang

Shandong University of Traditional Chinese Medicine

xinyu sun

Shandong University of Traditional Chinese Medicine

yingjie zhang

Shandong University of Traditional Chinese Medicine

\section{Research Article}

Keywords: Necroptosis, Ovarian cancer, Survival prediction, LncRNA

Posted Date: February 7th, 2022

DOI: https://doi.org/10.21203/rs.3.rs-1312197/v1

License: (9) This work is licensed under a Creative Commons Attribution 4.0 International License. Read Full License 
Necroptosis-associated LncRNA markers for late survival prediction, immune and gene mutation analysis in ovarian cancer

Wei Sen Fan ${ }^{1+}$, Ying Hua Qi ${ }^{2}$, Zhong Qi Fan ${ }^{1}$, Hao Ying Meng ${ }^{1}$, Ru Meng Yang ${ }^{1}$, Xin Yu Sun ${ }^{1}$, Ying Jie Zhang ${ }^{1 *}$

1.Qianfo Mountain Campus, Shandong University of Traditional Chinese Medicine, No. 16369 Jingxie Road, Lixia District, Jinan, Shandong 250013, China

2.Affiliated Hospital of Shandong University of Traditional Chinese Medicine, No. 42, West Culture Road, Lixia District, Jinan, Shandong, 250013, China

$\dagger$ : This symbol represents the first author

*: This symbol represents the corresponding author

\section{Abstract}

OBJECTIVE:To investigate the potential prognostic value of necroptosis-associated IncRNAs (NALncRNAs)in patients with ovarian cancer.

METHODS: Gene expression data from ovarian cancer patients with clinical data were downloaded from TCGA database. This is the base data for screening NALncRNAs that represent different survival times.IncRNA risk models were constructed by Cox regression analysis and evaluated for their prognostic value. The link between IncRNA signatures and related pathways, immune cell activity, immunological checkpoints, m6A-related genes, and gene mutations was also investigated.

RESULTS: In this study, eight NALnCRNAs including USP30-AS1, MINCR, LINC01096, DNM30S, LINC02574, DTNB-AS1, ACAP2-IT1, and ILVBL-AS1, all of which were well represented for the outcome of ovarian cancer patients. Noemogram after calibration curve validation has good clinical utility. Risk signatures were shown to be abundant in various pathways related to immunology and cell proliferation, according to gene set enrichment analysis. Patients' late survival outcome corresponds to differences in immune cells, immune checkpoints, immune function, etc.A better immune microenvironment would facilitate late survival, but inexplicably patients with long survival times have a higher immune escape. Patients with high mutations in genes appear to have better survival outcomes.

CONCLUSION: This study identified eight NALnCRNAs markers for the first time, providing a valuable basis for more accurate prediction of ovarian cancer prognosis.

Key words: Necroptosis; Ovarian cancer; Survival prediction; LncRNA

\section{Introduction}

Because of its more subtle onset, ovarian cancer is still the most difficult of the three major gynecologic cancers to diagnose, and roughly $70 \%$ of patients are diagnosed late, resulting in increased mortality and morbidity ${ }^{[1][1]}[2]$. Even after delicate treatment and care, there is still a possibility of recurrence[3]. Since the etiology and pathogenesis of ovarian cancer are not clear and survival time varies[4], it is essential that we search for new prognosis-related biomarkers for physicians to manage 
ovarian cancer patients at a later stage.

Necroptosis is a programmed form of cellular necrosis that can be initiated by tumor necrosis factor, interferon, and tumor necrosis factor-associated apoptosis-inducing antibodies[5]. One of the main hallmarks of tumors is resistance to apoptosis, so it is important to study apoptosis-related biomarkers for predicting or treating cancer[6]. currently, many studies have shown that the development of various cancers is associated with LncRNAs[7], and several LncRNAs have been identified as possible cancer indicators and targets[8][9].So we used bioinformatics from the perspective of necroptosis to search for LncRNAs that can determine the survival outcome of ovarian cancer.The detailed study route can be seen in Figure 1.

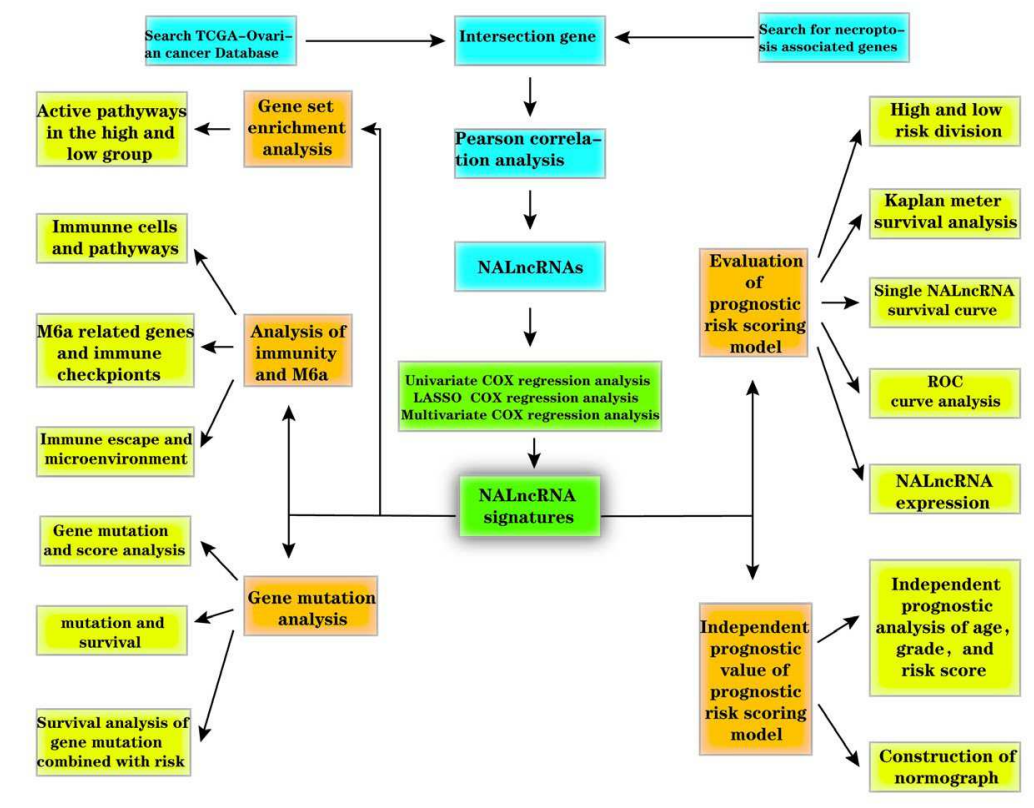

Figure1 flow chart of necrotizing apoptosis-related Incrnas in predicting ovarian cancer

\section{Methods}

\section{Data sources}

We obtained tumor tissue RNA sequence data (FPKM values) with clinical information from TCGA database (https://portal.gdc.cancer.gov/repository) for 379 ovarian cancer patients, and used R software ( 4.1.1) was used to extract and normalize the data. Two studies extracted necroptosis-associated genes(NAGs) from published reviews, and a third investigator was sought to decide when disagreement arose. The final result was 104 genes associated with necrotizing apoptosis and is provided in Annex Attachment 1. The intersection of the gene data in the expression matrices with the NAGs yielded the new NALncRNAs expression matrices. Since the TCGA database is an open access resource, this study was exempted from ethics committee approval.

\section{Identification of NALncRNAs}

To identify NALncRNAs in ovarian cancer patients, we used Pearson correlation to assess the degree of association between LncRNAs and NAGs. Typically, we used a 
threshold of $p<0.001$ and a correlation coefficient $\mid$ Correlation Coefficient $\mid>0.4$ to screen for NALncRNAs.

\section{Validation and construction of a prognostic risk model for NALncRNAs}

We used perl language (perl 5.28.1) to merge differentially expressed NALncRNAs expression with survival time, removing the mutilated and uninformative data. First, univariate Cox regression analysis was completed using the "survival" package to screen for necrotrophic apoptosis-related IncRNAs that could distinguish survival outcomes; We utilized the R package "glmnet" to limit the range screened by univariate and develop prognostic models using the least absolute shrinkage and selection operator (LASSO) Cox regression model to eliminate the influence of overfitting. Multivariate Cox regression analysis was used to determine which NALncRNAs predict survival in ovarian cancer patients.

Next from the already obtained necroptosis-related LncRNAs, the prognostic gene-LncRNA correlation network was drawn and visualized with Cytoscape software (3.7.2)to facilitate the observation of possible relationships between LncRNA and genes. Construct a scoring model based on survival outcomes of ovarian cancer patients and output risk score coefficients (coef) for genes included in the model. The constructed prognostic risk score model was calculated as: risk score $=$ prognostic NALncRNAs gene expression $1 \times$ coef1 + prognostic NALncRNAs gene expression $2 \times$ coef $2+\ldots+$ prognostic NALncRNAs gene expression $n \times$ coef $n$.The median determined by the above method is used to distinguish between high and low patient risk.

Kaplan-Meier analysis was used to compare the overall survival time (os) of the two groups, and HRs forest plots and heat maps of NALncRNAs prognostic models with survival curves for each prognostic gene were shown. Evaluate the relationship between each variable and risk value and prognosis using single-factor and multi-factor independent prognostic analysis to determine whether the constructed risk model can meet the criteria for independent prognosis. Evaluation of survival outcome prediction models using ROC curves with the "timeROC" package function of R software.

The samples were divided into groups based on their risk scores, and the risk score distribution and survival status were mapped using the R package "pheatmap." The Hosmer-Lemeshow test was also employed to see if the anticipated outcome was congruent with the actual outcome.

\section{Gene Set Enrichment Analysis(GSEA)}

Expressed gene sets from low- or high-risk populations and marker gene sets collected in the kegg database V7.4 were analyzed using GSEA (4.1.0) software. Setting FDR $<0.05$ is considered valuable.

\section{Immunocorrelation analysis}

We used CiberSort[10], TIMER[11], MCP-COUNTER[12], QUANTISEQ[13], XCell[14], and estimation of the ratio of immune cells to cancer cells (EPIC)[15] to assess the activity of immune cells in both populations based on FIRLS characteristics. Simultaneously, ssGSEA analysis was carried out using the R software package "gsva" to analyze the immune cell infiltration percentage and immune pathway activity in 
the high-risk and low-risk ovarian cancer groups. Immune checkpoints may be useful in guiding ovarian cancer treatment[16][17].The relationship between survival outcomes and immune checkpoints can be understood by analyzing gene expression levels in two groups of ovarian cancer patients with different survival times. Immune escape in ovarian cancer affects the effectiveness of immunotherapy[18], and the microenvironment of ovarian cancer cells interacts with tumor cells through secretory intercommunication, which can promote cancer cell invasion and metastasis[19][20]; therefore, we analyzed and compared the scores of immune escape and immune microenvironment in both groups.

\section{M6a gene correlation analysis}

M6A and LncRNA are linked to the development of ovarian cancer, particularly m6A-regulated CACNA1G-AS1, which has been identified as a cancerous LncRNA[21]. Because M6A-related genes are important regulators of tumor growth, we looked into the link between survival risk and m6a-related genes by comparing gene expression levels in two groups with varied survival outcomes.

\section{Gene mutation analysis}

Gene mutations play a role in the development of ovarian cancer[22] and its antagonistic therapy, and suitable regimens can be designed based on the tumor mutation profile. Different gene mutations have different effects[23] on the late stage of tumor patients, so we looked at gene mutations in high- and low-risk groups to see if there was a link between high and low mutation levels and ovarian cancer patients' survival times.

\section{Results}

\section{Identification of differentially expressed NALncRNAs}

Based on the most recent IncRNA annotation file, we found 4668 IncRNAs in the RNA-SEQ data of ovarian cancer patients (see Appendix S2), and then performed Pearson correlation analysis of these LncRNAs with 104 NAGs, resulting in 164 NALncRNAs, and finally identified 8 differentially expressed NALncRNAs.

\section{Construction of a NALncRNAs risk model with prognostic value}

In this study, data information from 379 ovarian cancer patients were selected for preliminary screening of prognosis-related LncRNAs using one-way Cox regression analysis, and a total of 12 necrotizing apoptosis-related LncRNAs associated with prognosis were identified (Figure .2A). Then, Lasso regression with multivariate Cox regression analysis was performed, and the results showed that 8 LncRNAs were included in the model (Figure 2B-D). These 8 prognostic NALncRNAs were USP30-AS1, MINCR, LINC01096, DNM3OS, LINC02574, DTNB-AS1, ACAP2-IT1, and ILVBL-AS1. Based on the best $\lambda$ value, a prognostic risk score model for NALncRNAs was constructed: risk value ( risk score) $=(-0.0784 \times$ PCAT19 expression value $)+$ $(1.0279 \times$ CDKN2B-AS1 expression value $)+(0.3622 \times$ LINC01936 expression value $)+$ (0.0099 $\times$ LINC02178 expression value $)+(-0.0065 \times$ BMPR1B-DT expression value $)+$ $(-0.1754 \times$ LINC00237 expression value $)+(-0.0801 \times$ TRPM2-AS expression value). The final resulting gene and LncRNA correlation network showed that 8 LncRNAs were associated with 14 genes. (Figure .2E) 


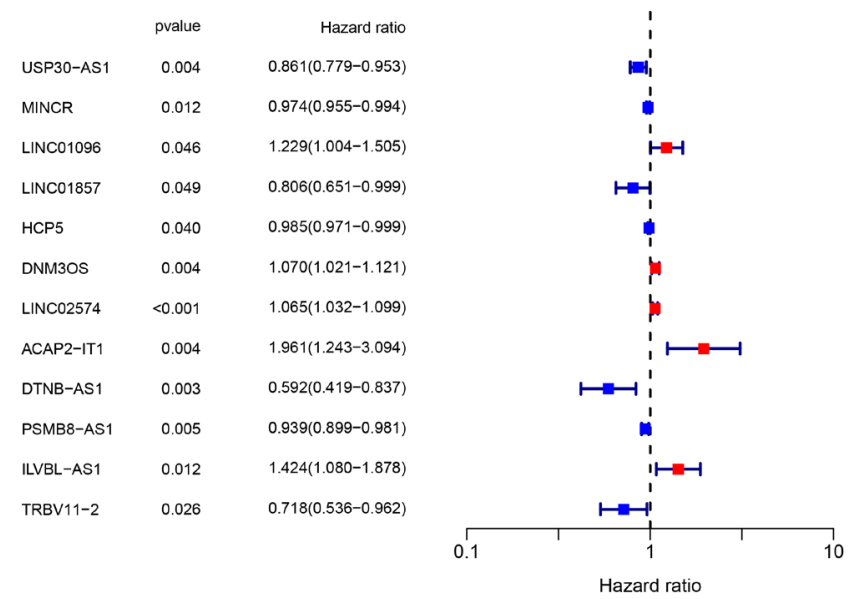

Figure .2A Univariate Cox regression analysis of 12 NALncRNAs initially screened for association with ovarian cancer prognosis

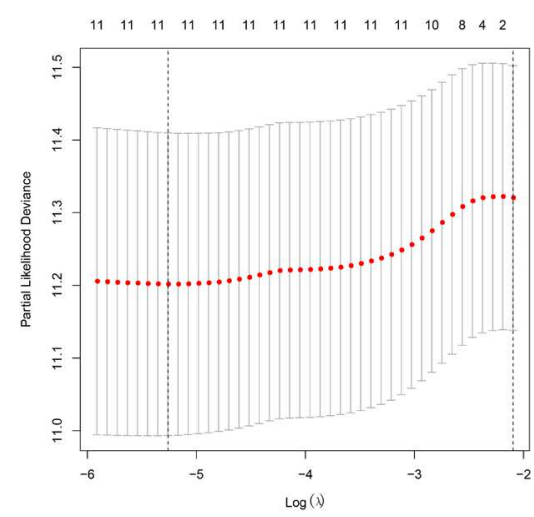

Figure .2B Multivariate cox regression forest plot shows that USP30-AS1、MINCR、 LINC01096、DNM3OS、LINC02574、DTNB-AS1、ACAP2-IT1 and ILVBL-AS1 were prognosis-associated NRLncRNAs and were involved in forming the prognostic model.



Figure .2C Lasso-Cox regression analysis shows that 12 NALncRNAs are good candidates for constructing prognostic characteristics. 


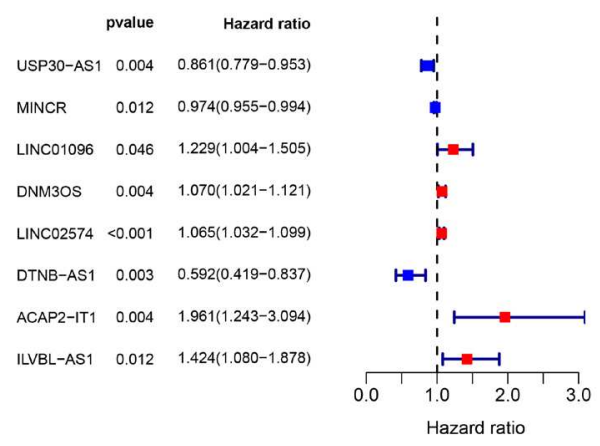

Figure .2D Multivariate cox regression forest plot shows that USP30-AS1、MINCR、 LINC01096、DNM3OS、LINC02574、DTNB-AS1、ACAP2-IT1and ILVBL-AS1 were prognosis-associated NALncRNAs and were involved in forming the prognostic model.

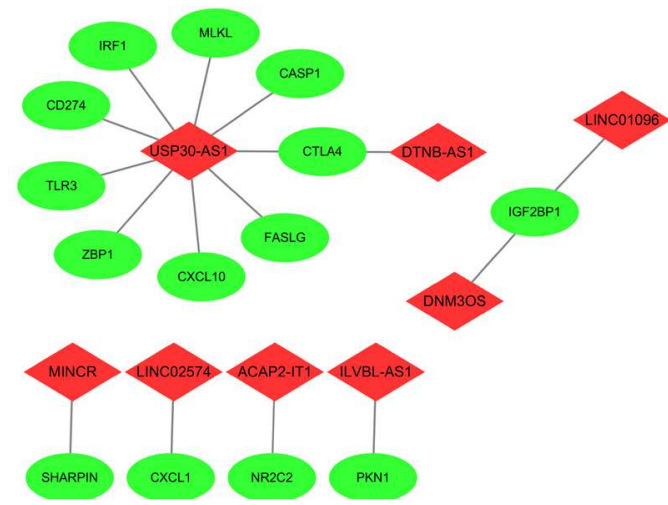

Figure .2E Gene-LncRNA co-expression network of prognosis-associated NAIncRNAs and NAGs.

\section{Prognostic risk score model evaluation}

The computed survival risk values were used to define the high- and low-risk groups of ovarian cancer patients included in the study. The risk score was proportional to the mortality rate(Figure $3 A-B)$. The high-risk group had a statistically significant lower survival rate than the low-risk group, according to k-M survival analysis, and the survival rate dropped year by year over time, with the high-risk group having a significantly lower survival rate than the low-risk group. (Figure 3C). Single-gene survival analysis was performed based on the expression profiles of eight prognostic genes, in which MINCR and USP30-AS1 were significantly different (Figure 3D). ROC curves were used to determine the sensitivity and specificity of the prognostic model, and the area under the curve at all four time points was more than 0.6, indicating that the risk model had good predictive power(Figure 3E).The heat map also clearly depicts the differences in expression of the eight NALncRNAs between the two risk groups. (Figure 4F) 


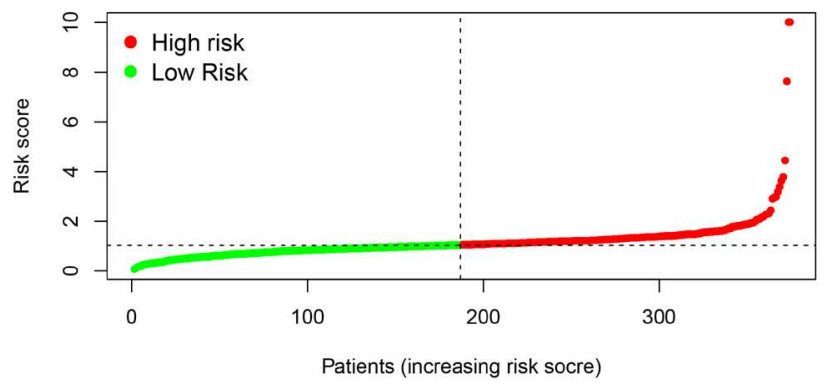

Figure .3A Differentiation of ovarian cancer patients based on risk score.

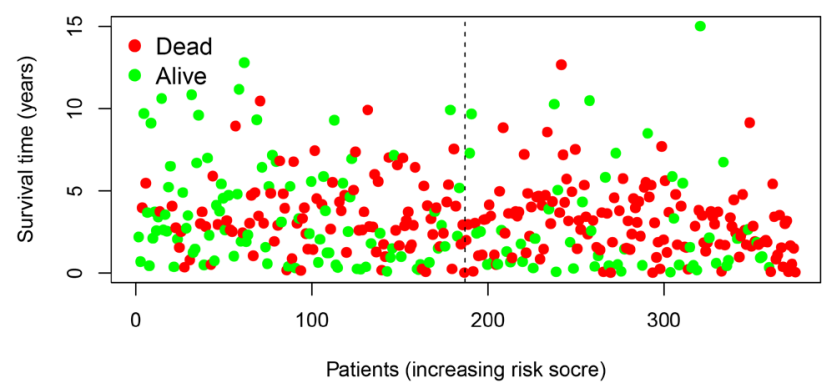

Figure .3B Patient's Survival Status Chart of ovarian cancer.

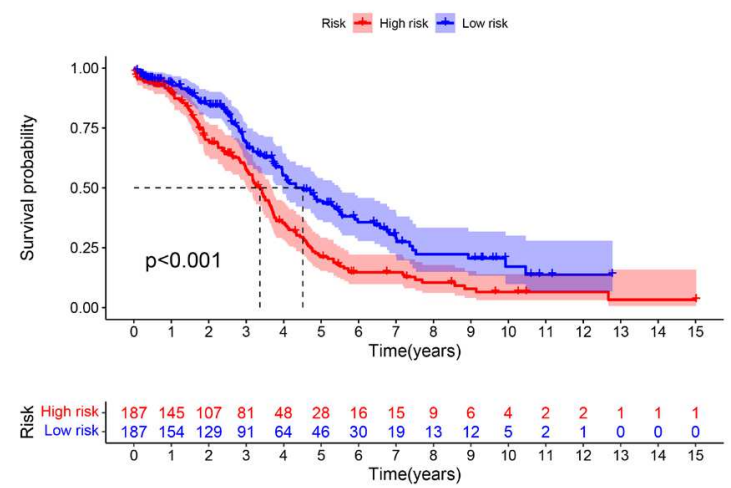

Figure $.3 \mathrm{C}$ Patients with varying ovarian cancer risks had dramatically variable survival durations, according to a Kaplan-Meier survival analysis.
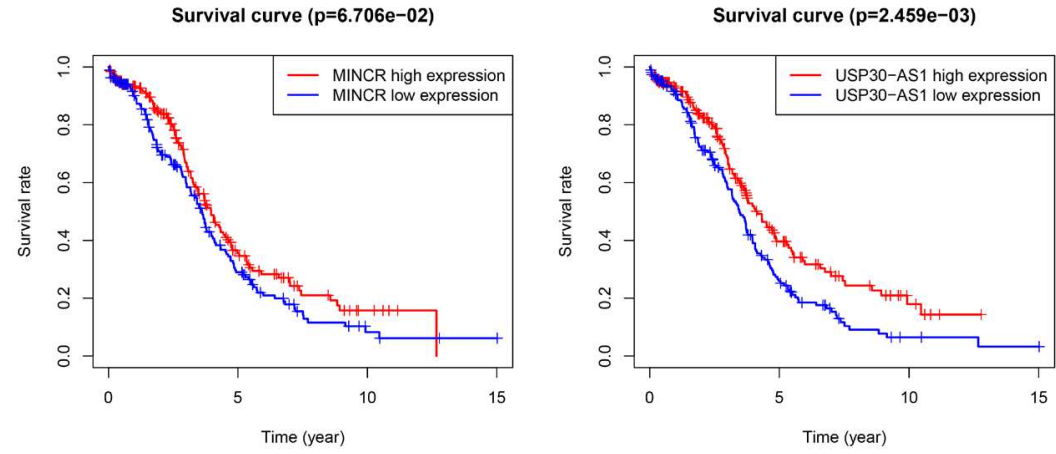


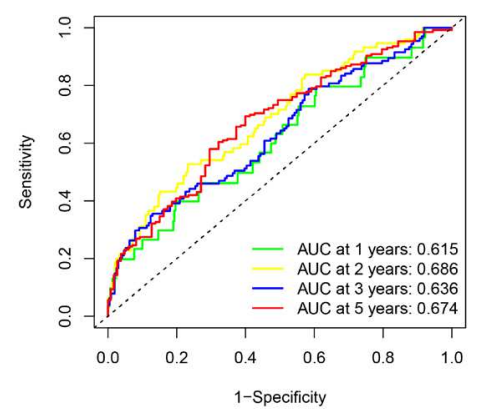

Figure .3EThe prognostic accuracy of the risk score was verified by the AUC values at four different time points.

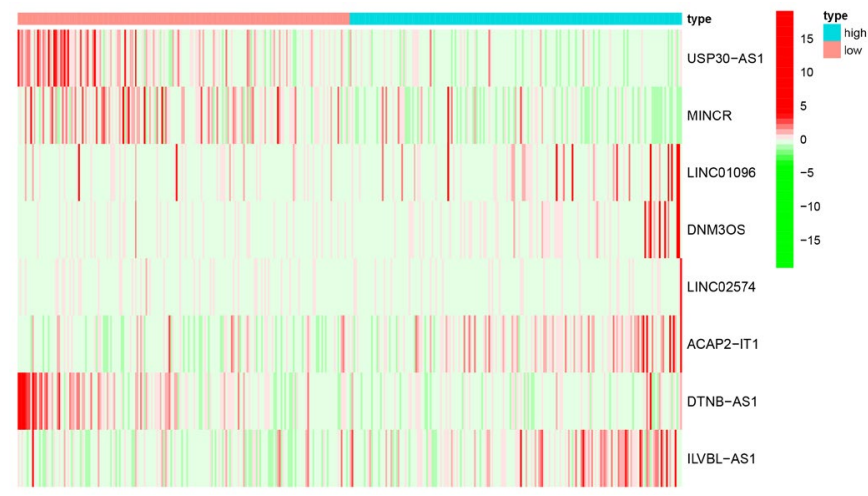

Figure.3F Eight selected NALncRNAs are significantly differentially expressed in ovarian cancer patients with different risks.

\section{The prognostic risk score model's independent prognostic value}

Univariate and multivariate Cox regression analyses were used to see if the prognostic risk score model could be used as an independent prognostic predictor. Both univariate and multivariate Cox regression analyses revealed that age and risk score were significantly associated with OS in ovarian cancer patients. (Figure 4A-B). As a result, age and risk score can be employed as independent prognostic indicators in the evaluation of ovarian cancer patients. the ROC curve illustrates the accuracy of these indicators as independent prognostic factors for patients (Figrue 4F), where grading is less accurate as an independent prognostic factor. Nomograms predict late survival outcomes based on patient age, classification, risk score and high and low risk(Figrue 4C). 1-year, 3-year, and 5-year corrected plots demonstrate the consistency of the predictions of the normograms with the observed outcomes. (Figrue 4D). 


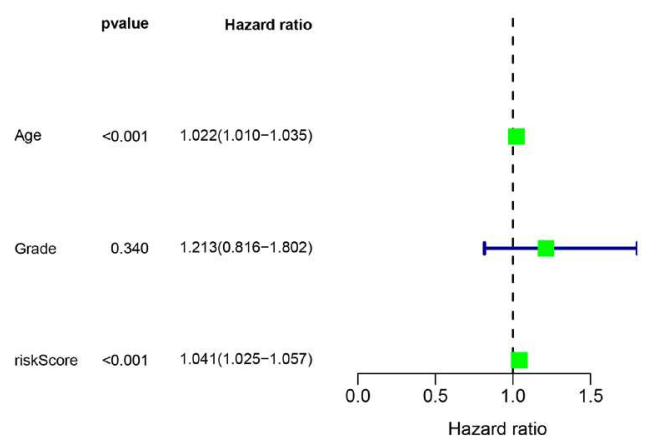

Figure.4A Age, grading, and risk score were used in a univariate independent prognostic analysis.

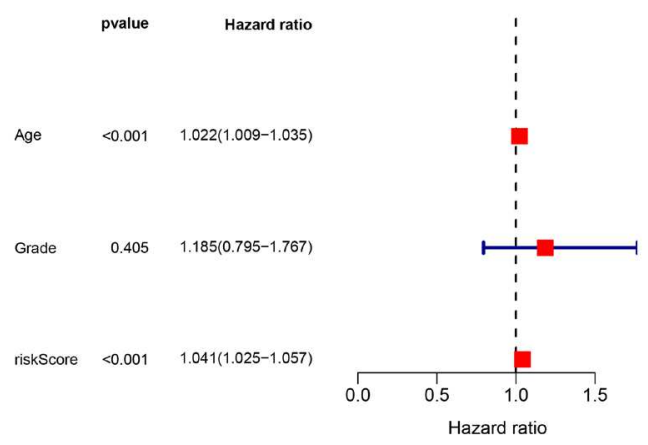

Figure.4B Age, grading, and risk score are all used in a multivariate independent prognostic analysis.

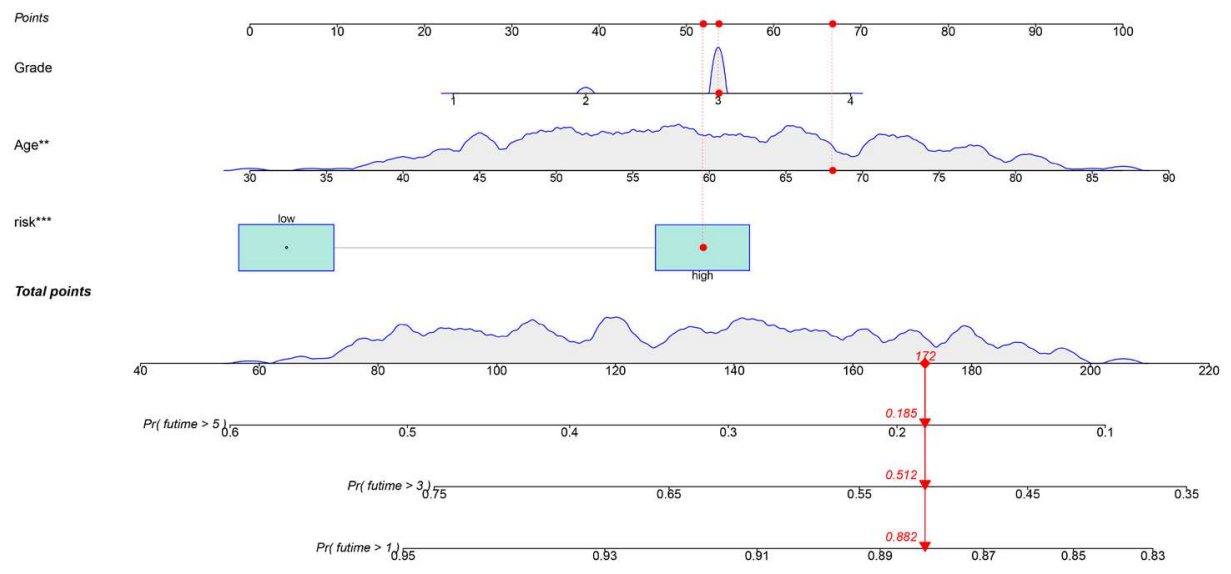

Figure.4C Nomogram combining risk scores with clinical factors 


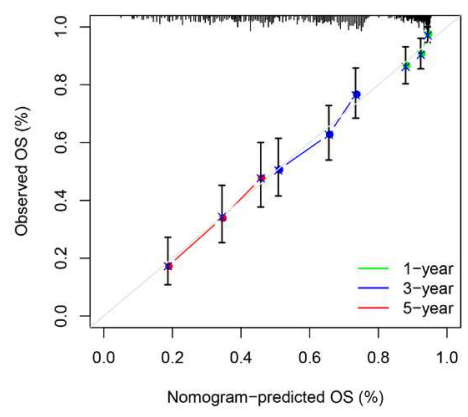

Figure.4D 1-year, 3-year and 5-year Nomogram correction curves

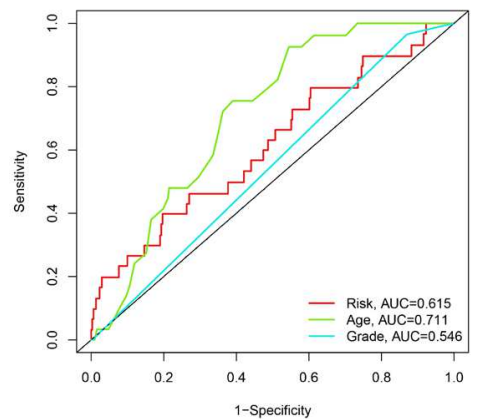

Figure.4F The AUC of the ROC curve validated the prognostic accuracy of prognostic-related clinical factors.

\section{Signaling pathway analysis}

A gene set enrichment analysis (GSEA) was done using differentially expressed genes between the high-risk and low-risk groups to investigate the biological activities and signaling pathways of NAlncRNAs.Endometrial cancer, MAPK signaling, ECM receptor, Endocytosis, and other related pathways were found to be more active in the high-risk ovarian cancer group. In the low-risk group, Peroxisome, Steroid hormone biosynthesis, Oxidative phosphorylation, Jak stat signaling, Arginine and proline metabolism, Alpha linolenic acid metabolism were more active. (Figure 5)

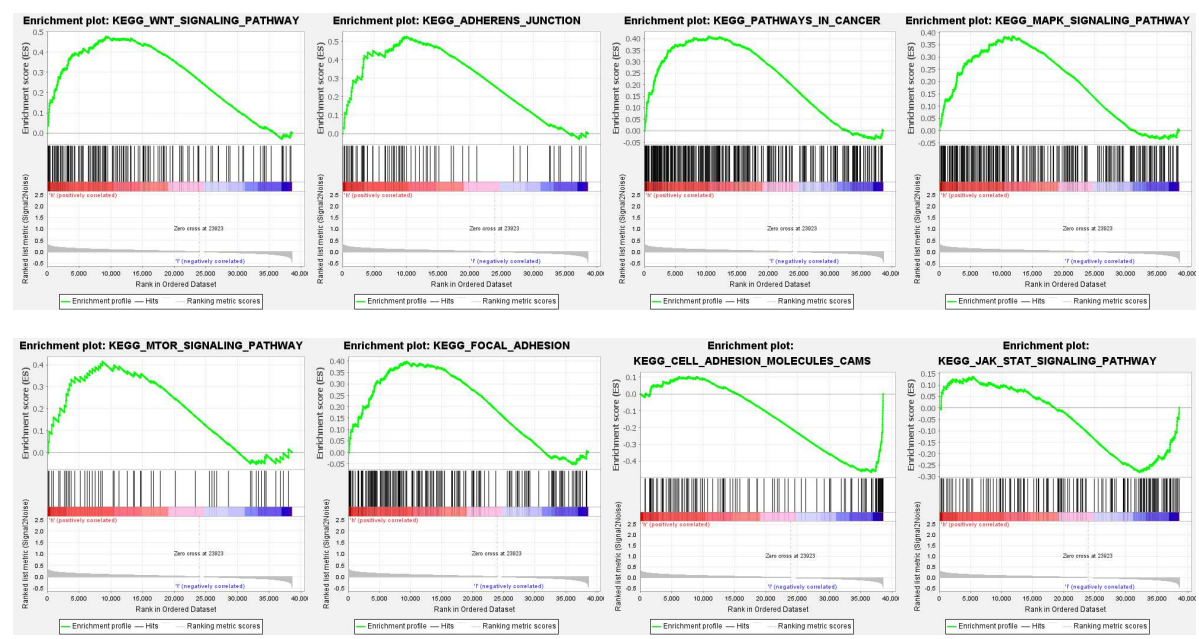




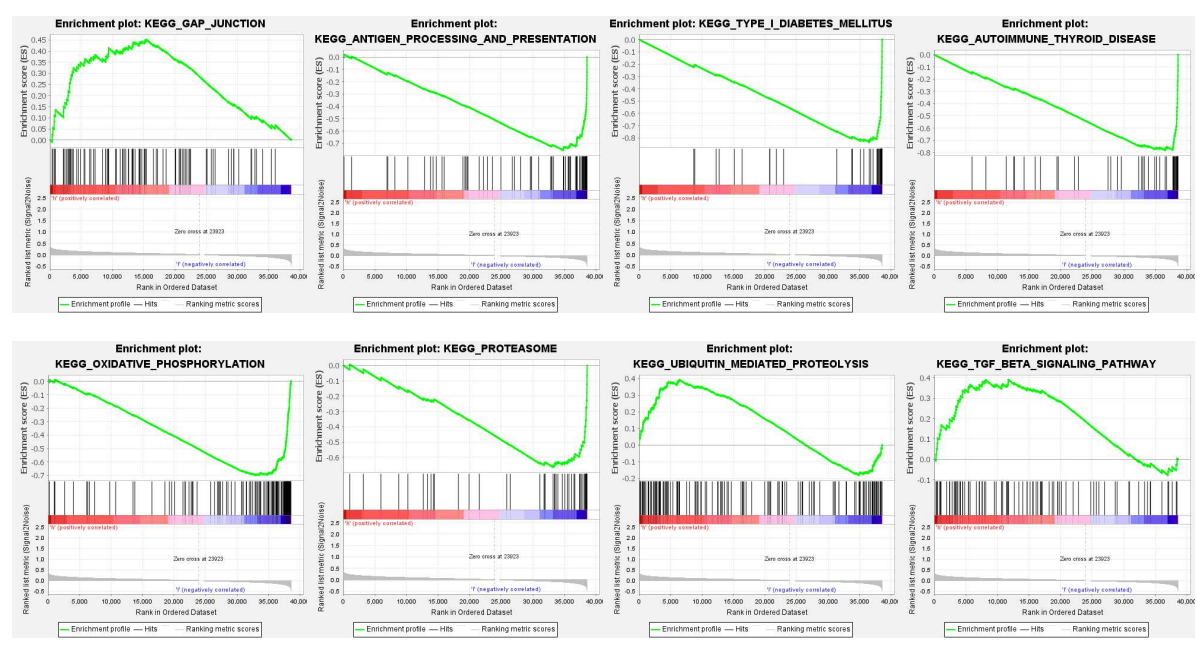

Figure.5The GSEA of NAGs differentially expressed in the high-risk and low-risk groups is presented by the pathway map. In the pathway map, the green curve is clearly raised or depressed in the different risk groups to represent that the pathway is active.

\section{Immune-related studies and m6a correlation analysis}

The immune-related heat map based on TIMER, CiberSort, QUANTISEQ, MCP-Counter, XCell and EPIC algorithms is shown in Figure 6A. Immunity, necroptosis, and tumor microenvironment are intertwined, and we compared the enrichment fractions and immune-related pathway activity of the more common immune cells using single-sample gene set enrichment analysis (SSGSEA)). (Figure 6B-C) and the results showed that between the two groups B_cells, aDCs,NK_cells, CCR, HLA and check-point were statistically significant. Given the relevance of checkpoint-based immunotherapy, there were also disparities in the expression of immunological checkpoints between the two groups.(Figure 6D), such as ICOS and LAG3. Because necroptosis is so important in the m6a process in human cells, there were changes in the expression of m6a-related genes between the two groups (Figure 6E), such as FTO and RBM15. the low-risk group had significantly higher differences in immune scores and total microenvironment scores than the high-risk group (Figure 6F). Surprisingly, immune escape scores were higher in the low-risk group than in the high-risk group. (Figure 6G)

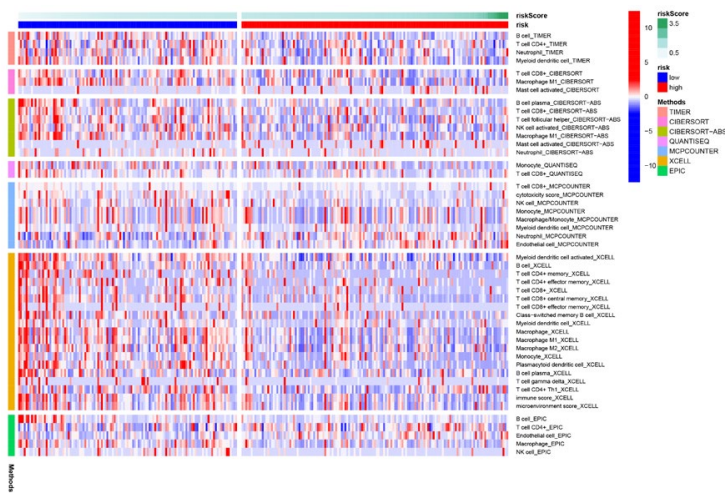

Figure.6A A multi-algorithm-based immune response heat map between low- and high-risk groups of ovarian cancer patients. 


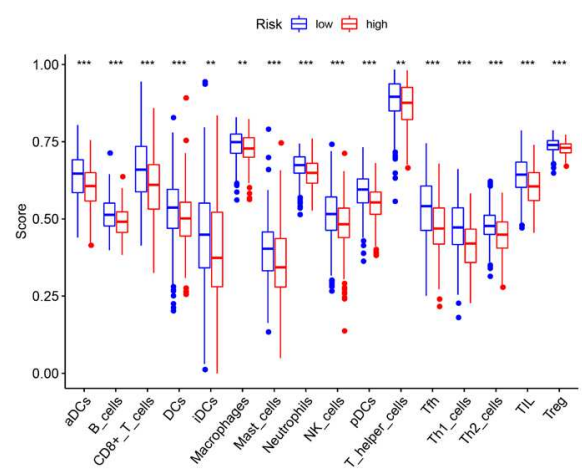

Figure.6B B-C Between low- and high-risk groups, ssGSEA scores of 16 immune cell types were compared to enrichment scores of 13 immune-related pathways.

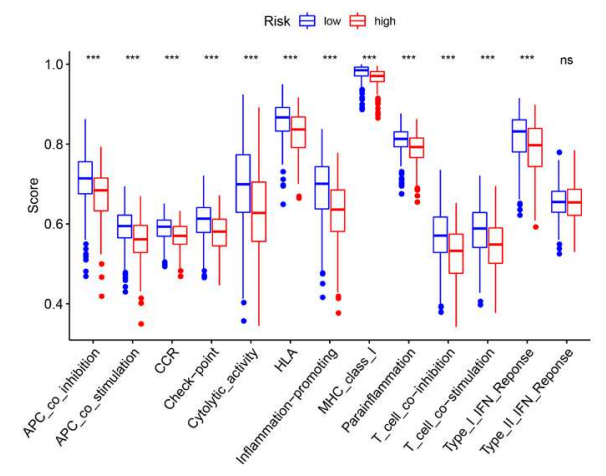

Figure $6 \mathrm{C}$

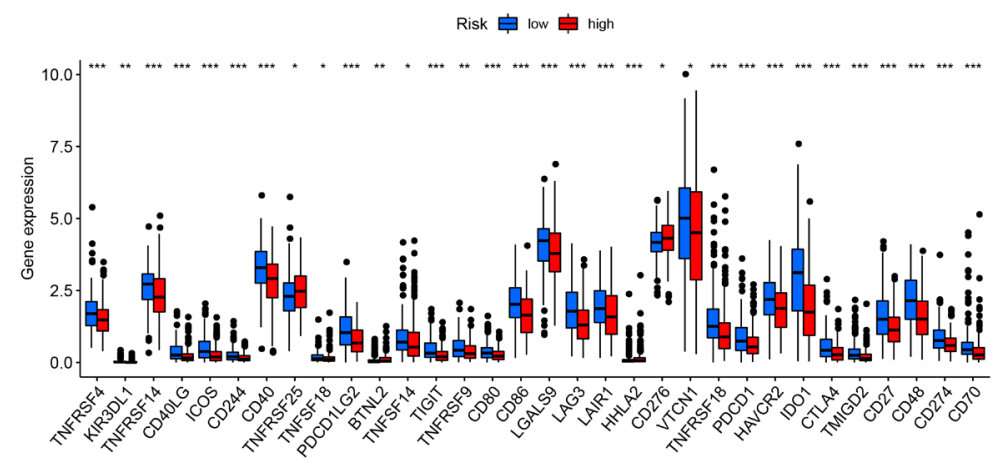

Figure.6D The difference in immune checkpoint expression between the two groups was investigated. 


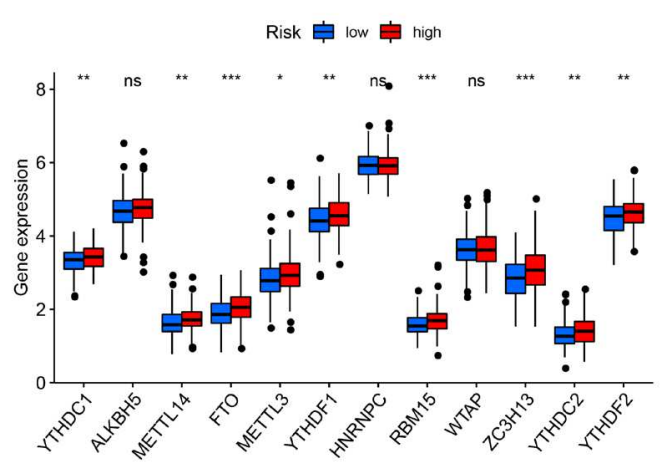

Figure.6E Associations between the risk signature and m6A-related genes.

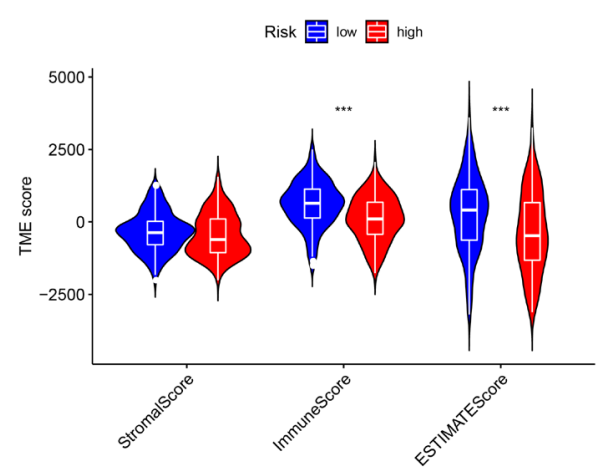

Figure.6F The high-risk and low-risk groups' stromal, immune, and ESTIMATE scores were plotted, and the low-risk group's immune and ESTIMATE scores were greater than the high-risk group's.

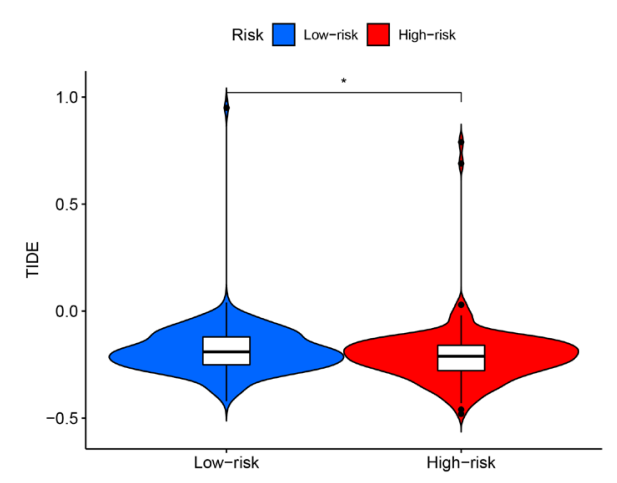

Figure.6G High and low risk groups had different immune escape scores, with the low risk group having higher TIDE scores than the high risk group.

\section{Gene mutation and survival prediction}

The analysis of the top 20 mutations in patients in the high and low risk groups showed that the mutated genes were the same in both groups and there was no difference in the mutation scores. (Figure 7A-C) Survival was higher in the high-mutation group than in the low-mutation group, with the best survival in the high-mutation-low-risk group and the worst survival in the low-mutation-high-risk group. (Figure 7D-E) 

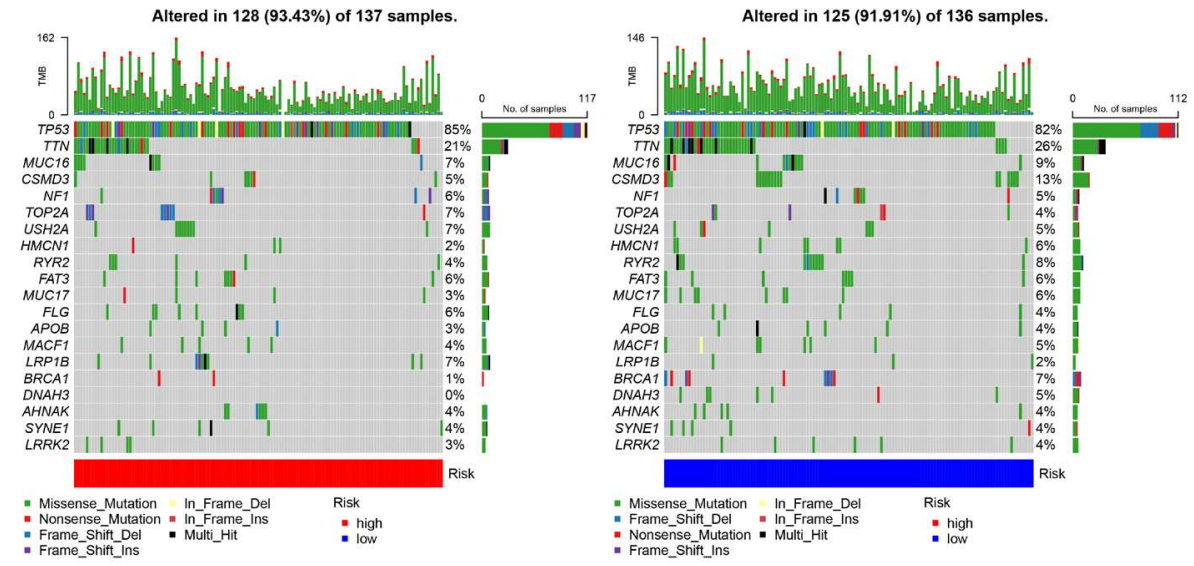

Figure 7A-B The percentages on the right in the waterfall plot of the top 20 mutations in the high and low risk groups represent the mutation frequency of the genes, and different colors represent different mutation types.

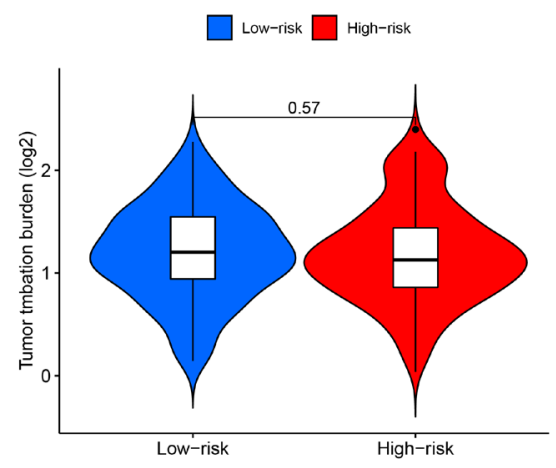

Figure.7C Analysis of gene mutation score, no difference between high and low risk groups.

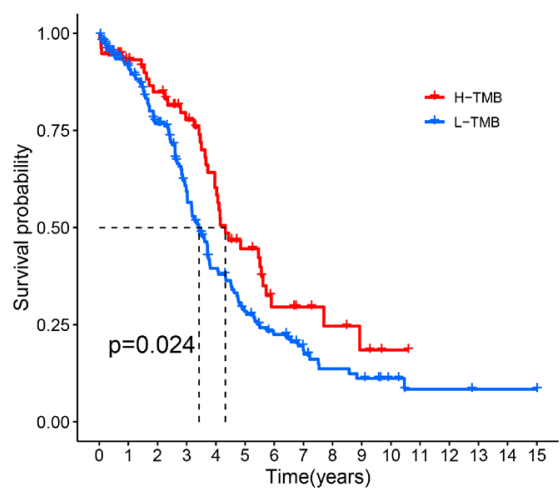

Figure.7D The graph of survival status difference between high and low mutation groups shows good survival status difference between high and low mutation groups. 


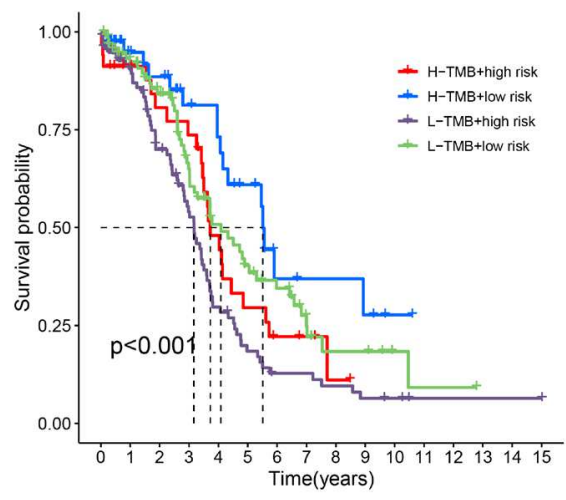

Figure.7E When the combined survival status of the high and low mutation groups, as well as the high and low risk groups, was analyzed, it was discovered that the high mutation low risk group had the greatest survival status, while the low mutation high risk group had the poorest.

\section{Discussion}

In recent years, there have been revolutionary advances in sequencing technology, and LncRNA, CircRNA and MiRNA have been detected. These do technology products can not only explain the onset and progression of cancer, but also provide research references for further treatment[24][25]. IncRNA plays a role in cell proliferation, differentiation, migration, invasion, and death via regulating gene transcription rate, translation, and post-translational modifications[26][27].Because the survival rate of ovarian cancer patients is among the lowest among gynecological cancers, this study looked into NALncRNAs to predict survival and further assessed the immunological and gene mutation status of high and low risk patients.

A total of 8 NALncRNAs were screened in this study, among which LINC01096, DNM3OS, LINC02574, ACAP2-IT1, and ILVBL-AS1 represented shorter survival time for ovarian cancer patients, and USP30-AS1, MINCR, and DTNB-AS1 represented longer survival time for ovarian cancer patients. Many studies have shown that the above LncRNAs are specific in the development of cancer.For example, in the current investigation, LINC01096 was not shown to be predictive for ovarian cancer but was found to be significantly expressed in breast cancer tissue cells, and knocking down LINC01096 prevented[28] cancer cell migration and invasion. DNM30S was overexpressed in ovarian cancer, and knockdown of it similarly inhibited migration and invasion of cancer cells and reduced mesenchymal to epithelial transformation[29].USP30-AS1 inhibits apoptosis in acute myeloid leukemia cells and may promote cancer cell survival through cis-regulation of USP30 and ANKRD13A[30]. By serving as a competitive endogenous RNA, MINCR may increase glioma cell proliferation and migration[31]. As seen in Figure.2E, IGF2BP1, LINC01096 and DNM3OS co-expression. It has been shown that IGF2BP1 promotes the invasion of SRC/MAPK-driven ovarian cancer cells[32].

Through GSEA, we found that pathways related to jak stat signaling, MAPK signaling, and endocytosis were more active in the high-risk group. jak stat signaling promotes tumor development and mediates almost all immunomodulatory processes, including immune escape[33]. MAPK signaling plays an important role in 
the proliferation and metastasis of ovarian cancer cells, and this pathway is usually interfered with to influence apoptosis of cancer cells[34]. The most important cause of death in ovarian cancer is recurrence and metastasis of tumor cells, and abnormal activation of the TGF- $\beta$ signaling pathway in active passages in the high-risk group is just able to promote tumor metastasis and recurrence[ 35 ]. Oxidative phosphorylation, arginine and proline metabolism, and alpha linolenic acid metabolism are more active pathways in the low-risk group of endometrial cancer. Among them, small molecule inhibitors often act on the multimeric complexes in the Oxidative phosphorylation pathway, thus inhibiting metabolism as well as inducing oxidative damage and cancer cell death[36]. Related investigators have shown that the arginine and proline metabolism pathway can be interfered with to induce apoptosis in ovarian cancer cells and inhibit S-phase growth[37].

The higher B cell enrichment scores in the low-risk group in the immune cell and pathway scores further imply that synergistic interactions between tumor-infiltrating $T$ cells and B cells may be linked to better ovarian cancer patient survival[38]. Upregulated expression of human leukocyte antigen-G (HLA-G) was one of the methods by which ovarian cancer cells avoided immune monitoring, and the HLA enrichment score was greater in the low-risk group.lt's perplexing that the low-risk group had a larger immune escape rate and a superior survival outcome deficiency[39]. In the immunological checkpoint, the low-risk group's IDO1 score was higher than the high-risk group's, and previous studies found a link between high IDO1 expression and overall ovarian cancer survival[ 40].We found that the expression of M6A-related genes FTO was higher in the high-risk group, and related studies showed that FTO could not only inhibit apoptosis but also promote proliferation of ovarian cancer cells[41]. Both immune scores and microenvironment scores were higher in the low-risk group of ovarian cancer in our findings, which also surface the positive role of better immune and microenvironment in survival outcomes in ovarian cancer patients.

In our mutation analysis study, the highest mutated gene in the high and low risk groups was TP53, which improves the accuracy of cancer survival prognosis[42]. The top 20 mutated genes were the same in both groups, and there was no difference in gene mutation scores. However, our findings suggest that high mutations are protective for ovarian cancer patients, and ovarian cancer patients with high mutations and low risk have the best survival, while those with low mutations and high risk have the worst survival.

\section{Limitations}

Although the LncRNA risk model we developed has strong predictive value in terms of LncRNA, immunity and gene mutation in high and low risk groups, there are some shortcomings in our current study. First, our data collection, although retrieving the original dataset used to build the IncRNA-related model from the TCGA database, still requires other databases to collect data to verify the accuracy of our results. Secondly, the exact mechanism by which necroptosis regulates the exact process of ovarian cancer is still unclear and needs to be elucidated by further experimental studies. 


\section{Conclusion}

In this study, eight NAIncRNA markers were identified for the first time, providing a valuable basis for more accurate prediction of prognosis in ovarian patients. And it provides a reference for further exploring the immune and gene mutation studies in ovarian cancer patients with high mortality.

Conflict of Interest: The author of this article declares that he or she has no conflicts of interest.

[1] Lim MC, Won YJ, Ko MJ, Kim M, Shim SH, Suh DH, et al. (2019). Incidence of cervical, endometrial, and ovarian cancer in Korea during 1999-2015.

J Gynecol Oncol. 30, e38.

[2] Zhang J, Chen Y, Chen X, Zhang W, Zhao L, Weng L, et al. Deubiquitinase USP35 restrains STING-mediated interferon signaling in ovarian cancer. (2021). Cel1 Death Differ. 28, 139-155.

[3] Gupta S, Nag S, Aggarwal S, Rauthan A, Warrier N. Maintenance therapy for recurrent epithelial ovarian cancer: current therapies and future perspectives - a review. (2019). J Ovarian Res. 12, 103.

[4] Bennetsen AKK, Baandrup L, Aalborg GL, Kjaer SK. Non-epithelial ovarian cancer in Denmark - Incidence and survival over nearly 40 years. (2020). Gynecol 0ncol. 157, 693-699.

[5] Khoury MK, Gupta K, Franco SR, Liu B. Necroptosis in the Pathophysiology of Disease. (2020). Am J Pathol. 190, 272-285.

[6] Carneiro BA, E1-Deiry WS. Targeting apoptosis in cancer therapy. (2020). Nat Rev Clin Oncol. 17, 395-417.

[7] Peng WX, Koirala P, Mo YY. LncRNA-mediated regulation of cel1 signaling in cancer. (2017). Oncogene. 36, 5661-5667.

[8] Liu Z, Mi M, Li X, Zheng X, Wu G, Zhang L. A 1ncRNA prognostic signature associated with immune infiltration and tumour mutation burden in breast cancer. (2020). J Ce11 Mo1 Med. 24, 12444-12456.

[9] Nie K, Deng Z, Zheng Z, Wen Y, Pan J, Jiang X, et al. Identification of a 14-1ncRNA Signature and Construction of a Prognostic Nomogram Predicting Overall Survival of Gastric Cancer. (2020). DNA Cel1 Biol. 39, 1532-1544.

[10] Chen B, Khodadoust MS, Liu CL, Newman AM, Alizadeh AA. Profiling Tumor Infiltrating Immune Cells with CIBERSORT. (2018). Methods Mol

Biol. 1711, 243-259.

[11] Newman AM, Liu CL, Green MR, Gentles AJ, Feng W, Xu Y, et al. Robust enumeration of cell subsets from tissue expression profiles. (2015). Nat Methods. 12, 453-7. 
[12] Becht E, Giraldo NA, Lacroix L, Buttard B, Elarouci N, Petitprez F, et al. Estimating the population abundance of tissue-infiltrating immune and stromal cell populations using gene expression. (2016). Genome Biol. 17, 218.

[13] Finote1lo F, Mayer C, Plattner C, Laschober G, Rieder D, Hackl H, et al. Molecular and pharmacological modulators of the tumor immune contexture revealed by deconvolution of RNA-seq data. (2019). Genome Med. 11, 34.

[14] Aran D, Hu Z, Butte AJ. xCe11: digitally portraying the tissue cellular heterogeneity landscape. (2017). Genome Biol. 18, 220.

[15] Racle J, Gfeller D. EPIC: A Tool to Estimate the Proportions of Different Cell Types from Bulk Gene Expression Data. (2020). Methods Mol Biol. 2120, 233-248.

[16] Wan C, Keany MP, Dong H, A1-Alem LF, Pandya UM, Lazo S, et al. Enhanced Efficacy of Simultaneous PD-1 and PD-L1 Immune Checkpoint Blockade in High-Grade Serous Ovarian Cancer. (2021). Cancer Res. 81, 158-173.

[17] Yang C, Xia BR, Zhang ZC, Zhang YJ, Lou G, Jin WL. Immunotherapy for Ovarian Cancer: Adjuvant, Combination, and Neoadjuvant. (2020). Front Immuno1. 11, 577869.

[18] Gao Y, Xu Y, Zhao S, Qian L, Song T, Zheng J, et al. Growth differentiation factor-15 promotes immune escape of ovarian cancer via targeting CD44 in dendritic cells. (2021). Exp Ce11 Res. 402, 112522.

[19] Luo Z, Wang Q, Lau WB, Lau B, Xu L, Zhao L, et al. Tumor microenvironment: The culprit for ovarian cancer metastasis? (2016). Cancer Lett. 377, 174-82. [20] Ghoneum A, Afify H, Salih Z, Kelly M, Said N. Role of tumor microenvironment in the pathobiology of ovarian cancer: Insights and therapeutic opportunities. (2018). Cancer Med. 7, 5047-5056.

[21] Zheng J, Guo J, Cao B, Zhou Y, Tong J. Identification and validation of 1ncRNAs involved in m6A regulation for patients with ovarian cancer. (2021). Cancer Cel1 Int.21, 363.

[22] Moufarrij S, Dandapani M, Arthofer E, Gomez S, Srivastava A, Lopez-Acevedo M, et al. Epigenetic therapy for ovarian cancer: promise and progress. (2019). Clin Epigenetics. 11, 7.

[23] Wang RQ, Chen CJ, Jing Y, Qin JY, Li Y, Chen GF, et al. Characteristics and prognostic significance of genetic mutations in acute myeloid leukemia based on a targeted next-generation sequencing technique. (2020). Cancer Med. 9, 8457-8467.

[24] Necula L, Matei L, Dragu D, Neagu AI, Mambet C, Nedeianu S, et al. Recent advances in gastric cancer early diagnosis. (2019). World J Gastroentero1. 25, 2029-2044.

[25] Santos RM, Moreno C, Zhang WC. Non-Coding RNAs in Lung Tumor Initiation and Progression. (2020). Int J Mol Sci. 21, 2774.

[26] Fang Y, Fullwood MJ. Roles, Functions, and Mechanisms of Long Non-coding RNAs in Cancer. (2016). Genomics Proteomics 
Bioinformatics. 14, 42-54.

[27] St Laurent G, Wahlestedt C, Kapranov P. The Landscape of long noncoding RNA classification. (2015). Trends Genet. 31, 239-51.

[28] Wang GP, Mou ZL, Xu YY, Liu GX, Wang DM, Zhang HP. LINC01096 knockdown inhibits progression of triple-negative breast cancer by increasing miR-3130-3p. (2019). Eur Rev Med Pharmacol Sci.23, 7445-7456.

[29] Mitra R, Chen X, Greenawalt EJ, Maulik U, Jiang W, Zhao Z, et al. Decoding critical long non-coding RNA in ovarian cancer epithelial-to-mesenchymal transition. (2017). Nat Commun. 8, 1604.

[30] Li Z, Xie X, Fan X, Li X. Long Non-coding RNA MINCR Regulates miR-876-5p/GSPT1 Axis to Aggravate G1ioma Progression. (2020). Neurochem Res. 45, 1690-1699.

[31] Bley N, Schott A, Müller S, Misiak D, Lederer M, Fuchs T, et al. IGF2BP1 is a targetable SRC/MAPK-dependent driver of invasive growth in ovarian cancer. (2021). RNA Biol. 18, 391-403.

[32] Bley N, Schott A, Müller S, Misiak D, Lederer M, Fuchs T, et al. IGF2BP1 is a targetable SRC/MAPK-dependent driver of invasive growth in ovarian cancer. (2021). RNA Biol. 18, 391-403.

[33] Owen KL, Brockwel1 NK, Parker BS. JAK-STAT Signaling: A Double-Edged Sword of Immune Regulation and Cancer Progression. (2019). Cancers

(Base1). 11, 2002.

[34] Liu Y, Ding Y, Nie Y, Yang M. EMP1 Promotes the Proliferation and Invasion of Ovarian Cancer Cells Through Activating the MAPK

Pathway. (2020). Onco Targets Ther. 13:2047-2055.

[35] Wen H, Qian M, He J, Li M, Yu Q, Leng Z. Inhibiting of self-renewal, migration and invasion of ovarian cancer stem cells by blocking TGF- $\beta$ pathway. (2020). PLoS One. 15, e0230230.

[36] Nayak AP, Kapur A, Barroilhet L, Patankar MS. Oxidative Phosphorylation: A Target for Novel Therapeutic Strategies Against Ovarian Cancer. (2018). Cancers (Base1). 10, 337.

[37] Gan C, Huang X, Wu Y, Zhan J, Zhang X, Liu Q, et al. Untargeted metabolomics study and pro-apoptotic properties of B-norcholestery1 benzimidazole compounds in ovarian cancer SK0V3 cells. (2020). J Steroid Biochem Mol Biol. 202, 105709.

[38] Hinchcliff E, Paquette C, Roszik J, Kelting S, Stoler MH, Mok SC, et al. Lymphocyte-specific kinase expression is a prognostic indicator in ovarian cancer and correlates with a prominent B cell transcriptional signature. (2019). Cancer Immunol Immunother. 68, 1515-1526.

[39] Sheu JJ, Shih IeM. Clinical and biological significance of HLA-G expression in ovarian cancer. (2007). Semin Cancer Biol. 17, 436-43.

[40] Niu N, Shen W, Zhong Y, Bast RC Jr, Jazaeri A, Sood AK, et al. Expression of B7-H4 and ID01 is associated with drug resistance and poor prognosis in high-grade serous ovarian carcinomas. (2021). Hum Pathol. 113, 20-27. 
[41] Zhao L, Kong X, Zhong W, Wang Y, Li P. FT0 accelerates ovarian cancer cell growth by promoting proliferation, inhibiting apoptosis, and activating autophagy. (2020). Pathol Res Pract. 216, 153042.

[42] Li VD, Li KH, Li JT. TP53 mutations as potential prognostic markers for specific cancers: analysis of data from The Cancer Genome Atlas and the International Agency for Research on Cancer TP53 Database. (2019). J Cancer Res Clin Oncol. 145, 625-636. 


\section{Supplementary Files}

This is a list of supplementary files associated with this preprint. Click to download.

- attachment1.txt

- attachment2.txt 